Classification

Physics Abstracts

61.16 $\mathrm{Di}-61.50 \mathrm{Jr}-61.70 \mathrm{r}$

This article is the same as the one published in Microscopy, Microanalysis, Microstructures, vol. 2 (1991) $n^{\circ} 6$, from p. 627 to p. 636.

It is published again due to the bad quality of the figures.

\title{
Experimental image processing of small supported metallic particles (1-3 nm)
}

\author{
Suzanne Giorgio $\left({ }^{1}\right)$, Geneviève $\operatorname{Nihoul}\left({ }^{2}\right)$, Claude Chapon $\left({ }^{1}\right)$ and Claude R. Henry $\left({ }^{1}\right)$ \\ (1) CRMC2 (*) CNRS, Campus de Luminy Case 913, 13288 Marseille Cedex 9, France \\ (2) GMET, Groupe Materiaux, Université de Toulon, BP 132, 83957 La Garde Cedex, France
}

(Received January 13, 1992; accepted February 18, 1992)

\begin{abstract}
Résumé. - De petites particules métalliques cristallisées sur des cubes de $\mathrm{MgO}$ ont été observées de profil par MEHR. Les images de haute résolution sont traitées numériquement pour améliorer les contrastes de la morphologie des particules et des défauts éventuels à l'interface métal/MgO. Trois filtres différents, appliqués dans l'espace de Fourier, ont été testés. Un filtre qui conserve les fréquences spatiales voisines des réflexions (200), réhausse le contraste des particules par rapport au bruit de fond. Des filtres de Bragg autour des réflexions (200) mettent en évidence les défauts de la structure. Un nouveau filtrage, par soustraction du bruit de fond, améliore à la fois le contraste des particules et les défauts de la structure à l'interface.
\end{abstract}

\begin{abstract}
Metallic particles crystallized on $\mathrm{MgO}$ cubes have been observed in profile view in HRTEM. The images are processed in order to improve the visibility of the particles morphology and the eventual defects at the interface metal/MgO. Three different filters have been used, all of them working in the Fourier space. A band pass filter keeping all the spatial frequencies close to the (200) reflections improves the contast of the particles against the noise. Bragg filters around the (200) reflections show up the lattice defects. A new filtering by subtraction of the background, improves both the contrast of the particles and the details at the interface.
\end{abstract}

\section{Introduction.}

Small metallic particles deposited on oxide single crystals are often used as model catalysts [1]; their crystalline structure is usually determined by HRTEM. If the observation of particles larger than $5 \mathrm{~nm}$ does not present major difficulties, problems arise with smaller clusters for which high irradiation dosis are able to modify the structure [2-4]. A carbon coating on the samples prevents from structural fluctuations but introduces noise in the images.

Imaging of particles on $\mathrm{MgO}$ micro-cubes [4-6], allows the observation both in top view and in cross section of the particles and the direct visualization of the interface metal/oxide. However,

$\left(^{*}\right)$ Laboratoire propre du CNRS associé aux universités d'Aix- Marseille II et III. 
the contrast of the particles and of the interface is buried in the Fresnel fringes at the edge of the support.

Image processing of the HRTEM images is used in order to extract both the morphology of the small particles seen in profile view (limit between the crystalline material and the coating) and the defects at the interface metal/oxide. A large variety of common image processings are commercialized, most of them working in real space; for periodic objects [7-9], it is more convenient to apply filters in the Fourier space and to obtain processed image by inverse Fourier transform. Image processing of HRTEM pictures with non periodic features has been investigated [10] in the case of metallic interfaces and boundaries. Non periodic objects introduce continuous components around the main spots, which have to be kept. Specific filters in the Fourier space were already tested according to their ability to preserve the non periodic details in the image (defects,...). Two types of filters are often selected: a circular band pass filter in the Fourier space, preserving the interesting frequencies, removes some of the noise and the Fresnel fringes [11], while a Bragg filter, must be chosen if one is interested in particular details in the lattice.

Another type of Fourier space processing has been proposed [12], where all the points intensities lower than a given threshold are reduced to zero. This type of filter does not introduce as many ghost lattices as do the Bragg filters but it can suppress the non periodic details which correspond to an often weak diffuse area in the Fourier transform.

Finally, in the case of small metal particles, the single value decomposition method [13] was applied: though it only enhances one direction of fringes, the morphology of the particle was better seen.

In this paper, we want to compare different manners of processing HRTEM images of small $\mathrm{Au}$ and Pd particles, seen in profile view on MgO cubes.

\section{Experimental images.}

2.1 SAMPLE PREPARATION AND OBSERVATION IN HRTEM. - A Mg ribbon, burnt in pure oxygen and nitrogen in a vacuum chamber, produced $\mathrm{MgO}$ cubes collected on a microscope grid covered with a carbon film [5]. The grid was brought in front of a Knudsen cell for the vacuum metal deposition. Then, a carbon film of average thickness $3 \mathrm{~nm}$, was deposited on the sample to avoid further contamination and to stabilize the particles in the electron beam.

The samples were observed with a Jeol 4000 EX working at $400 \mathrm{kV}$. Figure 1 shows the general view of microcubes covered with small Au particles $(<5 \mathrm{~nm})$ sitting on their top ans side surfaces. Here, the $\mathrm{MgO}$ cubes are seen along their $<100>$ axis so that the (200) planes are imaged in HRTEM.

2.2 PRocessing of THE HRTEM IMAges: RING AND BRAgG FILTERS. - For the small particles having a low contrast, as in figures $2 \mathrm{a}, \mathrm{b}, \mathrm{c}$, the morphology has to be precised by filtering the noise arising from the carbon films. The high resolution images were digitized with 256 grey levels and $256 \times 256$ pixels with a sampling of 1 pixel for $0.02 \mathrm{~nm}$ at the level of the object, so that two neighbouring lattice fringes are separated by about 10 pixels. Their Fourier transforms (FT) (Figs. 3a, b, c) show the central beam and the four strongest (200) reflections. All the reflections are elongated in the direction normal to the interface. The (200) reflections are separated from the central beam by 21 pixels; one pixel in the Fourier space represents then $0.2 \mathrm{~nm}^{-1}$.

An example of the radial distribution in the FT is given in figure $3 \mathrm{~d}$. There, the intensity between the zero spacial frequency and the (200) reflections does not fall to zero and contains oscillations due to the presence of the amorphous background. Also, the peak corresponding to the (200) reflections is enlarged. 


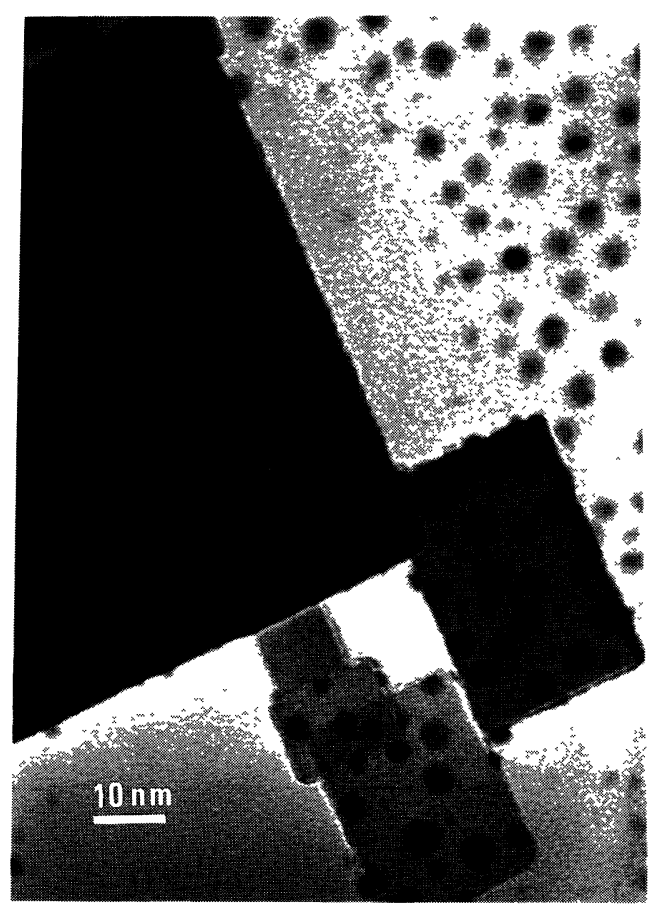

Fig. 1. - Overview of $\mathrm{MgO}$ cubes with particles on their surface and on their edges.
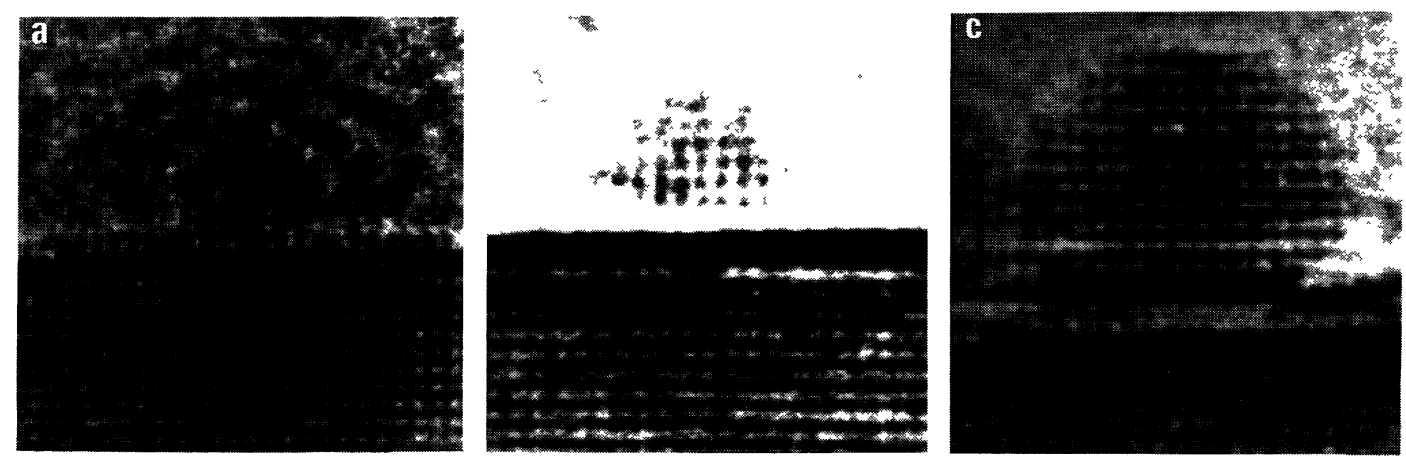

Fig. 2. - a) Small palladium particle $(1 \mathrm{~nm})$ seen in profile view, b) gold particle $(1.6 \mathrm{~nm})$, c) gold particle $(3.6 \mathrm{~nm})$.

A ring filter centered on the zero frequency (band pass filter in the Fourier space), having as its mean radius the (200) reflections frequency was applied; several widths were tried. In order to avoid the artefacts produced by discontinuous edges, a filter with soft edges was chosen, first with a Gaussian shape, then with a shape described by equation (1), giving a flatter maximum and sharp edges:

$$
f(g)=\exp \left(-\left(g-g_{0}\right)^{4} / \sigma^{4}\right)
$$



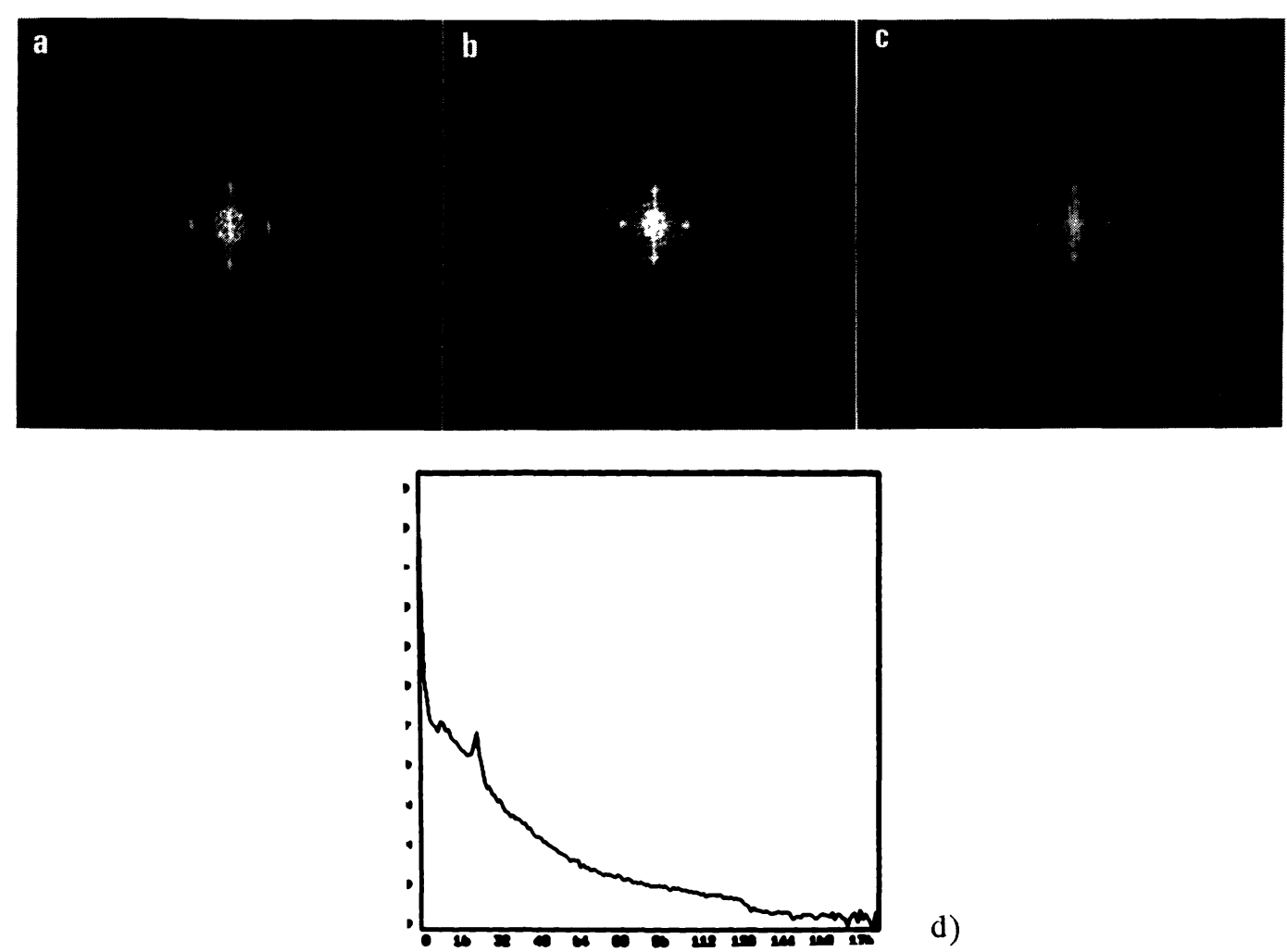

d)

Fig. 3. - a,b,c, Fourier Transforms of the images (Figs. 2a,b,c). d) Logarithm of the radial distribution of the Fourier transform (3b).

where $g$ is the mean radius of the filter $\left(g_{0}=g(200)\right)$ and $\sigma$ is a parameter defining the average width of the ring filter.

Figures $4 a, b, c$ give processed images of figures $2 a, b, c$ with $\sigma=3,4,5,6$ pixels corresponding to $\sigma=0.7,0.9,1.1$ and $1.3 \mathrm{~nm}^{-1}$ respectively in the Fourier space.

The processed images of the 1-2 nm sized particles (Figs. 2a, 2b) seem to change between the smallest values of $\sigma(=3)$ and $\sigma=4,5,6$. Indeed, the top of the particles is difficult to localize for $\sigma=3$. The contrast of the particles compared to the background decreases for increasing values of $\sigma$ while the non periodic details (the morphology, the eventual distorsions in the image at the interface) appear more clearly. Then, ghost fringes parallel to the interface and to the upper edge are found for the lowest values of $\sigma$. In fact, for the smallest particles (Fig. 2a) having $N=5$ cells along the interface, the enlargement $1 /(N * a)$ of the reflections in the Fourier space is 0.95 $\mathrm{nm}^{-1}$, so that the non periodic details might be completely lost by the filters with $\sigma<4.3$. On the opposite, in the image of the large particles (Fig. 2c) with 17 cells and a stronger contrast, the visualization of the morphology is not significantly improved, however for all particles, the details hidden in the Fresnel fringe now appear with the four values of $\sigma$.

In order to remove the artefacts in the FT, introduced by a limited square digitizing [14] (streaks parallel to the window edges), the digitized image was multiplied by a circular mask $\mathrm{C}$ with soft edges. Then, in the FT (5b), of image $2 b$ which has been multiplied by the mask $C(5 a)$, the 


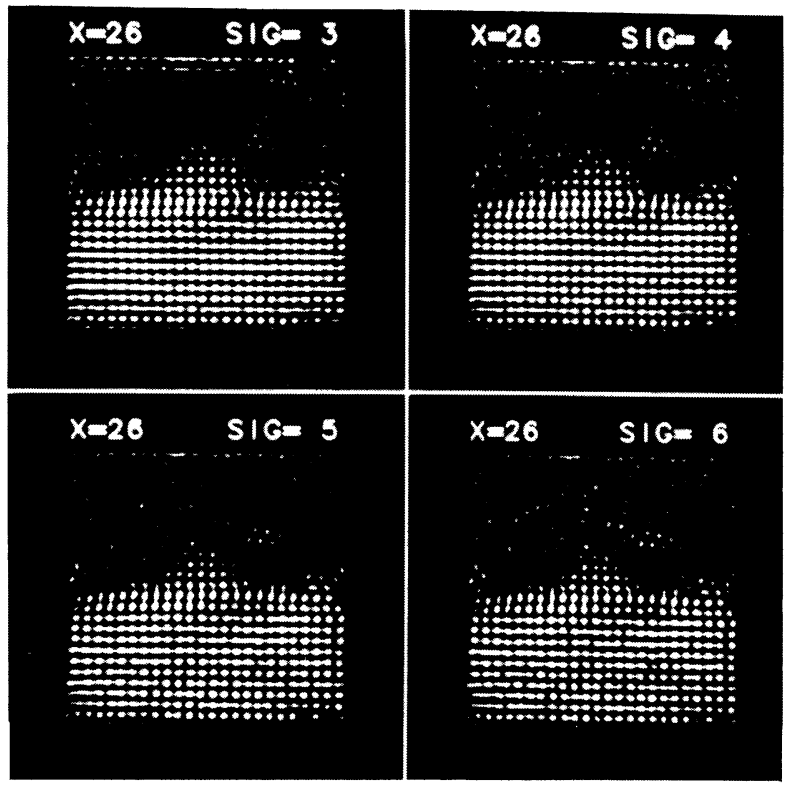

a)

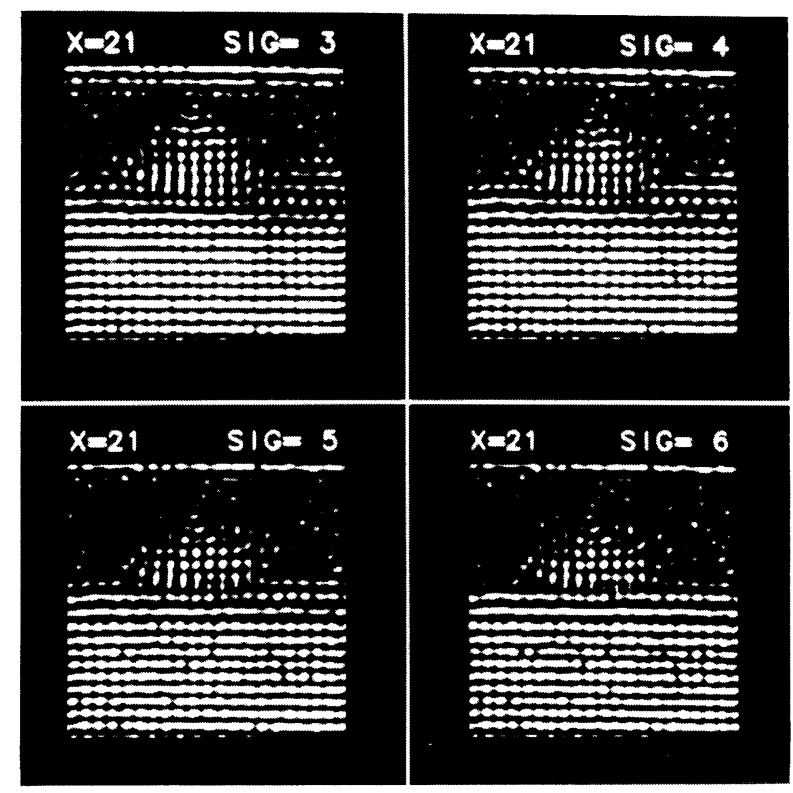

b)

Fig. 4. - a, b, c Processed images from figures $2 \mathrm{a}, \mathrm{b}, \mathrm{c}$ by noise filtering with ring filters with increasing widths ( $\sigma=3,4,5,6$ respectively). $X$ indicates the average radius of the ring. 

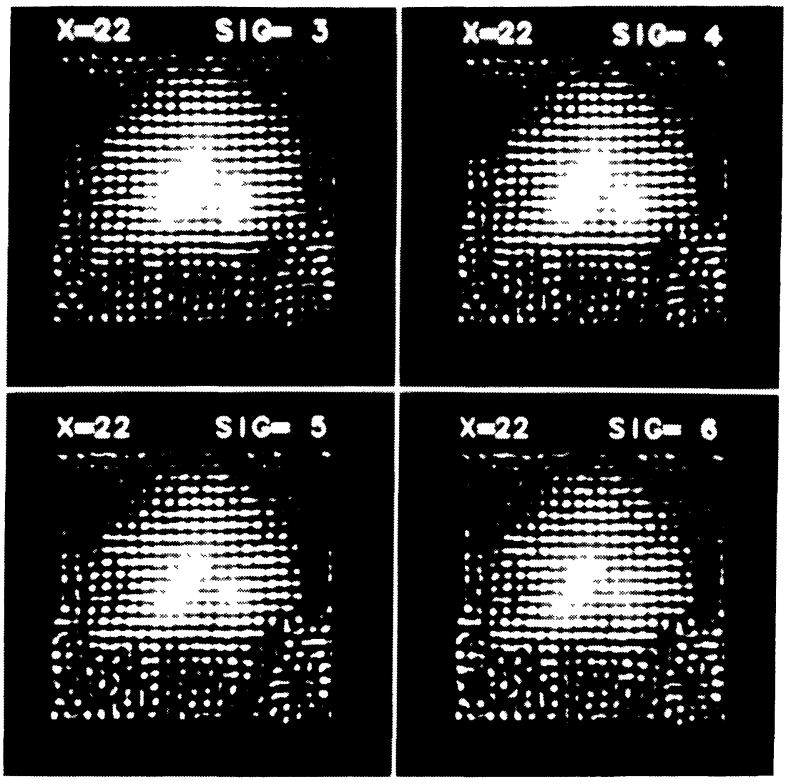

c)

Fig. 4. - (continued)

a)
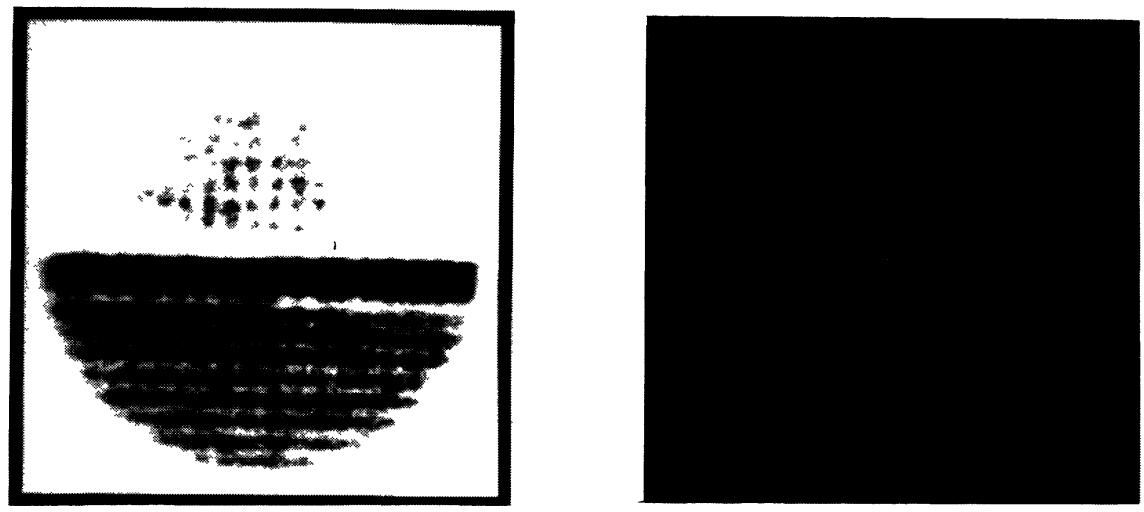

b)

Fig. 5. - a) Image $2 b$ masked with the ring filter. b) Fourier transform of $5 a$.

lengthening of the spots due to the square window are less pronounced (compare $5 \mathrm{~b}$ to $3 \mathrm{~b}$ ).

Then, the eventual ghost lattices found in figures $4 \mathrm{~b}$ near the edges deseappear from the background (see Figs. 6a, b, c, d).

The same particles have been processed by Bragg filters with circular holes centered on two or four (200) reflections. The profile of each hole with soft edges is given by equation (2):

$$
f(\mathbf{g})=\exp \left(-\left(\mathbf{g}-\mathbf{g}_{0}\right)^{2} / \sigma^{2}\right)
$$

where $\mathbf{g}$ is a vector of the Fourier transform of the image, and $\mathrm{g}_{0}$ is a (200) vector. 

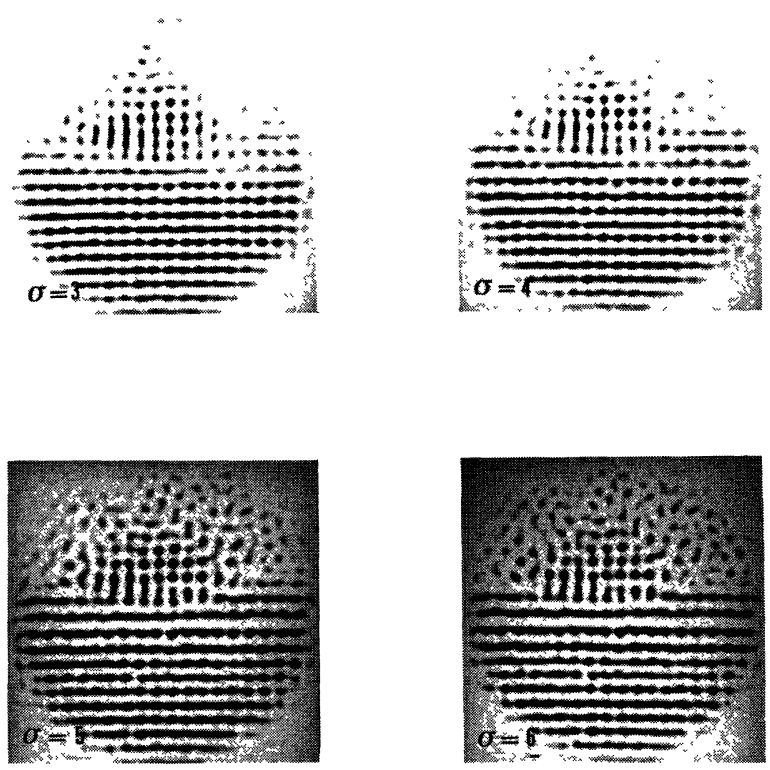

Fig. 6. - a, b, c, d Processed images from $2 b$ with the ring mask (5a), applied on the real image, and with ring filter applied on the Fourier transform, $(\sigma=3,4,5,6$, respectively).

Figures 7a, $\mathrm{b}, \mathrm{c}$ show the particle from figure $2 \mathrm{~b}$ processed with these Bragg filters for increasing values of $\sigma$. Ghost lattices are created in the amorphous area, as could be expected as the radial distribution of the FT (Fig.3d) showed a high contribution of the amorphous background, around the (200) spatial frequency.

Defects are shown by processing with only two Bragg filters corresponding to two opposite (200) reflections (Figs. 7a, b, c). For increasing widths of the disc $\left(\sigma^{2}=4,16,25\right)$, imperfections in the lattice appear. Some strong distortions can be sometimes seen in the crystalline area, but also in the ghost lattices in the background. In fact, the amorphous background is able to give discontinuities in the frequencies, then artefacts are seen in the backgound of the processed image. As the particles and the $\mathrm{MgO}$ are enclosed between two carbon layers, the same distortions may be superimposed on the crystal. So, this type of treatment must be used very carefully in the case of a mixture of amorphous and crystalline superimposed material. However, it is the most powerful method to enhance the defects in a crystalline area.

2.3 PROCESSING BY SUBTRACTION OF THE BACKGROUND. - When looking at the radial distribution of the FT, it is tempting to try to subtract the background due to the amorphous part from the whole signal in order to only retain the crystalline contribution. The background for the spatial frequencies around the (200) reflections can be calculated by averaging the values for pixels corresponding to the same spatial frequencies but far from the (200) spots.

Two circles, centered on the central beam and including the (200) reflections were drawn. This ring was separated in three parts, the middle including the reflections, the inner and the outer ones were used to calculate the intensities of the background $I_{1}$ and $I_{3}$. The background in the middle ring $I_{2}$ was given the average values $\left(I_{1}+I_{3}\right) / 2$. Then, the points located outside the four circles were given the intensity zero and the intensity of each point in the circles was compared to $I_{2}$. If the intensity was smaller than $I_{2}$, the point was taken as background and the intensity was 


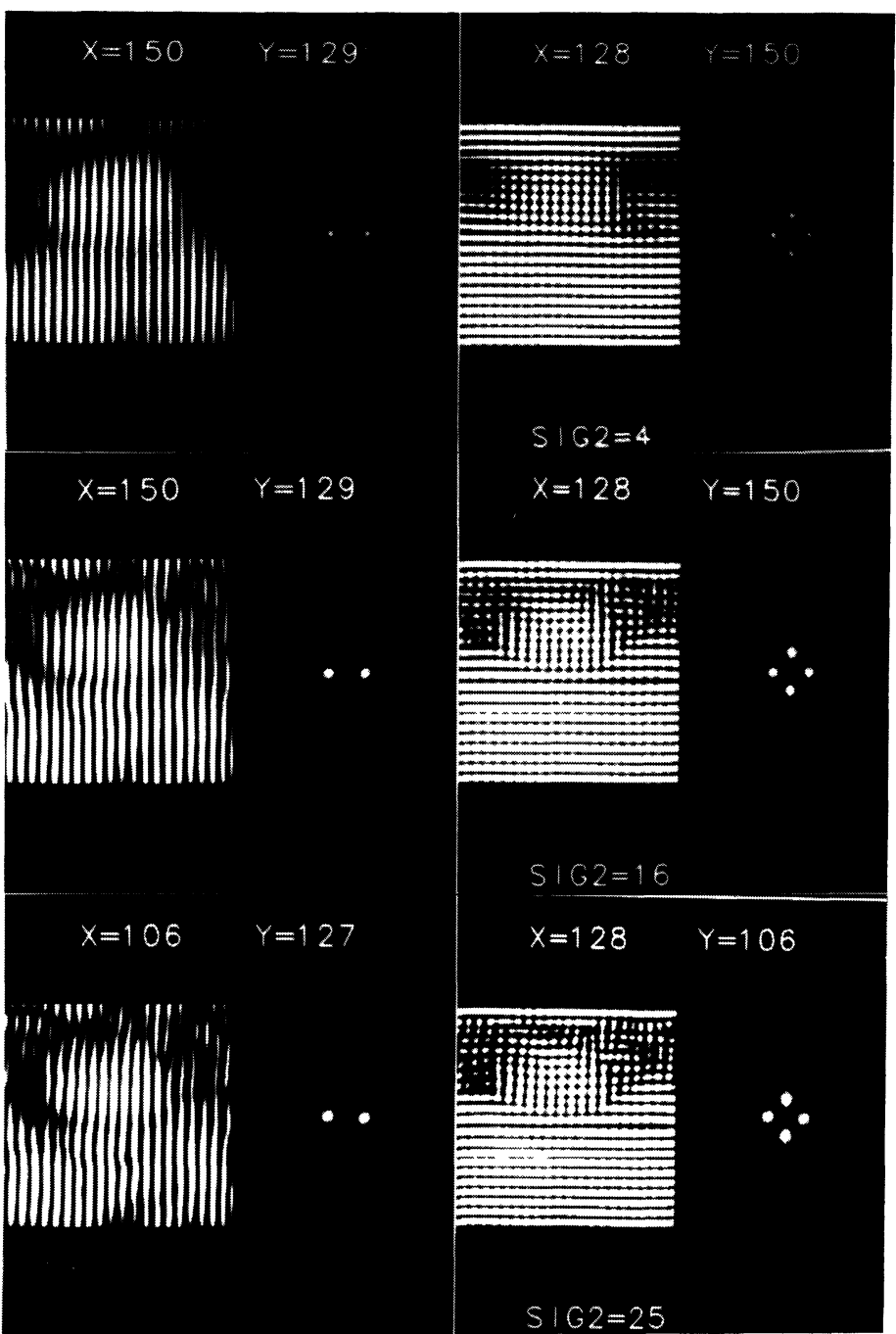

Fig. 7. - a, b, c, Processed images from figure $2 \mathrm{~b}$ with the Bragg filter (one pair and two pairs of holes) with increasing values of $\sigma(\sigma=4,16,25) . X$ and $Y$ are the coordinates of the centers of the discs, SIG2 is the square of the filter width $\sigma$.

put to zero. In the other case, it was considered as part of a reflection and two processings were tried:

i) the intensity of the point was unchanged, (procedure A).

ii) the average intensity of the background $I_{2}$ was subtracted from the intensity of the point, (procedure B).

In both procedures, $A$ and $B$, the phase was kept.

This method is more detailed in the theoretical part, [15].

The results of this processing on the images shown ir: figures $2 \mathrm{a}, \mathrm{b}, \mathrm{c}$, are shown in figure 8 , for four different mask widths $(\sigma=6,8,10,12)$.

The best images are obtained for the largest widths values (bottom right), which show that we 
need enough pixels to get a proper average. As shown in the theoretical par [15], it is better to use circles with a diameter equal to the crown width. There is not much difference between the two procedures $\mathrm{A}$ and $\mathrm{B}$, however, the contrast of the crystalline part is enhanced in $\mathrm{B}$. Compared to the images obtained by the ring filter processing, the interface between the particles and the oxide substrate can be clearly seen.
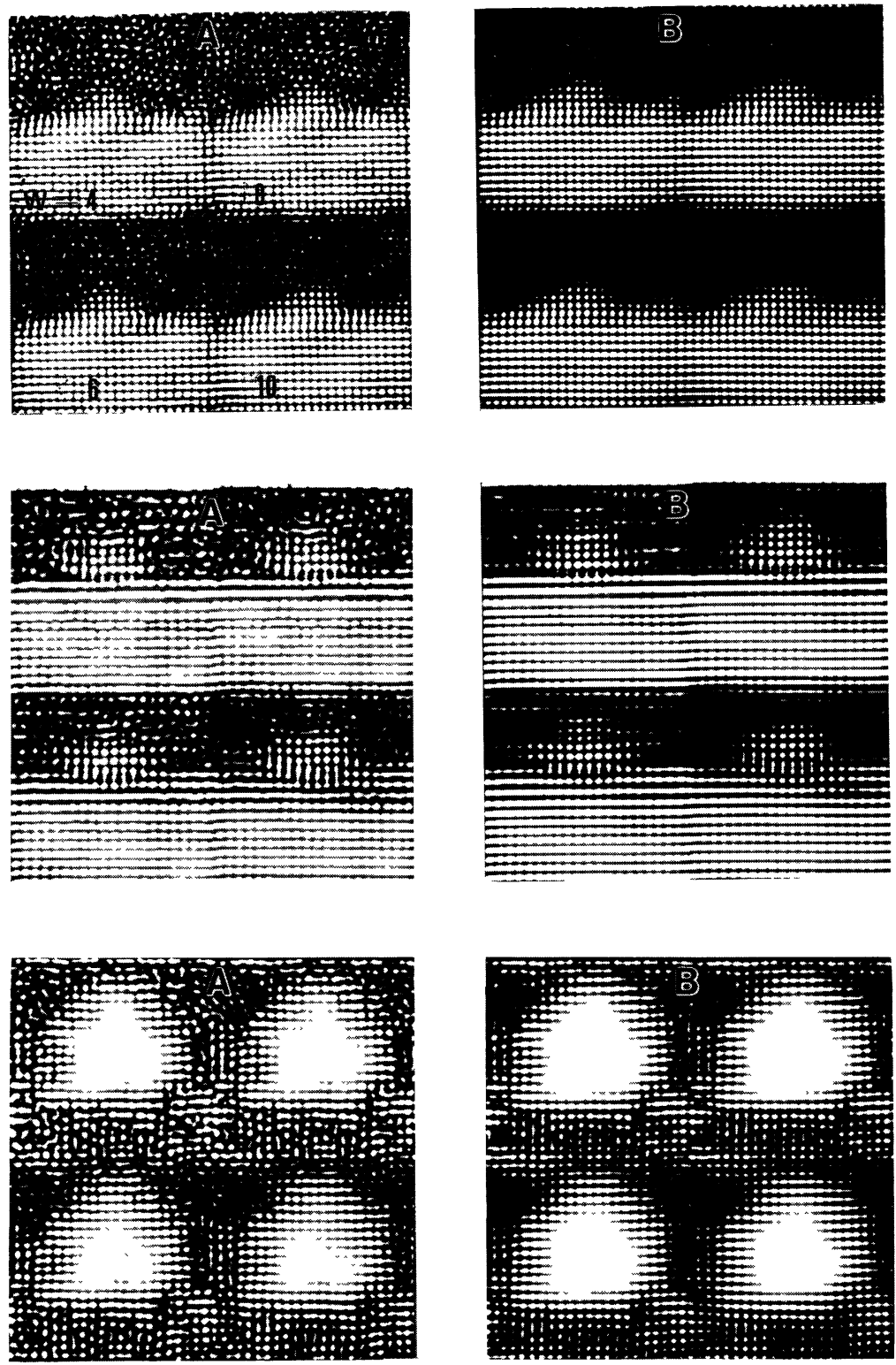

Fig. 8. - a,b,c Processed images from figures $2 a, b, c$ respectively, by subtraction of the background (left colum, by the procedure A, right column by $B$ ). 


\section{Dicussion.}

The application of three different image processings, working in the Fourier space, have shown interesting details in HRTEM profile images of supported small metallic particles.

- The band-pass filter improves the particles morphology by subtraction of the frequencies different from the ones around the (200) reflections. This type of mask also extracts the details of the interface, normally hidden in the Fresnel fringes.

- The Bragg filter enhances the defects and distortions at the interface, but introduces ghost lattices in the background. This type of filter is difficult to be used to process images where a crystalline area and an amorphous background are superimposed. However, it is a powerful treatment to underline the structural defects in crystals.

- The subtraction of the amorphous background gives results comparable to the band-pass filter as it improves the morphology of the particles and the details at the interface but it enhances more the contrast between the crystalline and amorphous areas.

In order to keep the non periodic details, one must take into account that the particles have a limited size, so that their reflections are enlarged in the FT and the width of the masks has to be calculated according to each particle size.

\section{References}

[1] Henry C.R., Chapon C. and Duriez C., Z. Phys. D 19 (1991) 347.

[2] IIJIMA S. and ICHIHASHI T., Phys. Rev. Lett. 56 (1986) 616.

[3] AJAYAN P.M. and MARKS L.D., Phys. Rev. Lett. 63 (1989) 279.

[4] Giorgio S., HenRY C.R., ChaPON C., Nihoul G., Penisson J.M., Ultramicroscopy 38 (1991) 1.

[5] Giorgio S., Henry C.R., ChaPON C., Penisson J.M., J. Cryst. Growth 100 (1990) 254.

[6] Giorgio S., Chapon C., Henry C.R., Nihoul G., Penisson J.M., Philos. Mag. A 64 (1991) 87.

[7] HAWKES P.W., Optik 40 (1974) 539.

[8] SAXTON W.O., Computer Techniques for image processing in electron microscopy (New York, Academic press, 1978);

SAXTON W.O., Computer processing of electron microscope images, P.W. Hawkes Ed. (Springer Verlag Berlin, New York, 1980) p. 15.

[9] HAWKES P.W., Computer graphics and image processing 18 (1982) 58.

[10] de JONG A.F., COENE W. and VAN DYCK D., Ultramicroscopy 27 (1989) 53.

[11] SAXTON W.O., in Proceeding of the 44th annual meeting of the electron microscopy Society of America, G.W. Bailey Ed. (San Francisco Press, 1986) pp. 526-529.

[12] PRADÈRE P. and ThOMAS E.L., Ultramicroscopy 32 (1990) 149.

[13] ARTal P., Avalos BorJa M., SORIA F., POPPA H., Heinemann K., Ultramicroscopy 30 (1989) 405.

[14] TOMTTA M., HASHIMOTO H., IKUTA T., ENDOH H. and YOKOTA Y., Ultramicroscopy 16 (1985) 9.

[15] NiHOUL G., Microsc. Microanal. Microstruct. 2 (1991) 637. 